\title{
Fixed Point Approximation for a Class of Generalized Nonexpansive Mappings in Hadamard Spaces
}

\author{
Kifayat Ullah $\mathbb{D}^{1,2}$ Junaid Ahmad ${ }^{1,},{ }^{3}$ Akbar Ali Khan, ${ }^{1}$ and Manuel de la Sen $\left(\mathbb{D}{ }^{4}\right.$ \\ ${ }^{1}$ Department of Mathematics, University of Science and Technology, Bannu, 28100 Khyber Pakhtunkhwa, Pakistan \\ ${ }^{2}$ Department of Mathematics, University of Lakki Marwat, Lakki Marwat, 28420 Khyber Pakhtunkhwa, Pakistan \\ ${ }^{3}$ Department of Mathematics, International Islamic University, H-10, Islamabad 44000, Pakistan \\ ${ }^{4}$ Institute of Research and Development of Processes, University of the Basque Country, Campus of Leioa (Bizkaia) P.O. Box 644- \\ Bilbao, Barrio Sarriena, 48940 Leioa, Spain \\ Correspondence should be addressed to Junaid Ahmad; ahmadjunaid436@gmail.com
}

Received 9 December 2020; Revised 19 April 2021; Accepted 3 May 2021; Published 15 June 2021

Academic Editor: Antonio Scarfone

Copyright (C) 2021 Kifayat Ullah et al. This is an open access article distributed under the Creative Commons Attribution License, which permits unrestricted use, distribution, and reproduction in any medium, provided the original work is properly cited.

In this paper, we establish strong and $\Delta$ convergence results for mappings satisfying condition $\left(B_{\gamma, \mu}\right)$ through a newly introduced iterative process called JA iteration process. A nonlinear Hadamard space is used the ground space for establishing our main results. A novel example is provided for the support of our main results and claims. The presented results are the good extension of the corresponding results present in the literature.

\section{Introduction}

Recall that a selfmap $T$ on a subset $E$ of a metric space is called nonexpansive if

$$
\rho\left(T a^{\prime}, T a^{\prime \prime}\right) \leq \rho\left(a^{\prime}, a^{\prime \prime}\right) \text { for every } a^{\prime}, a^{\prime \prime} \in E \text {. }
$$

Once a member $w$ is available in the set $E$ such that $w$ $=T w$ then it is called a fixed point of $T$. In this research, the notation $F_{T}$ will throughout represent the fixed point set of $T$. The study of fixed points for nonexpansive operators is a crucial and busy research field now a days. One of the ealier result of Gohde [1] states that in the frame work of uniform convexity of Banach space, nonexpansive operators always admits a fixed point on closed bounded and convex subsets. Kirk [2,3] was the first, who initiated fixed point theory of nonexpansive operators in the framework of nonlinear CAT(0) spaces. In 2008, Suzuki [4] achieved a big breakthrough by introducing a weak notion of nonexpansive operators. Notice that a selfmap $T$ of a subset $E$ of a metric space is said to satisfy Condition $(C)$ (also called Suzuki map) if for any $a^{\prime}, a^{\prime \prime} \in E$, we have

$$
\frac{1}{2} \rho\left(a^{\prime}, T a^{\prime}\right) \leq \rho\left(a^{\prime}, a^{\prime \prime}\right) \Rightarrow \rho\left(T a^{\prime}, T a^{\prime \prime}\right) \leq \rho\left(a^{\prime}, a^{\prime \prime}\right)
$$

The class of Suzuki nonexpansive mappings in linear and nonlinear setting were extensively studied by many researchers [5-12]. Very recently in 2018, Patir et al. [13] suggested a two parametric condition, which they called it Condition $B_{\gamma, \mu}$. They proved that the Condition $B_{\gamma, \mu}$ is weaker than the corresponding condition $(C)$. Recently, Varatechakongka and Phuengrattana [14] studied Condition ( $\left.B_{\gamma, \mu}\right)$ in the setting of Hadamard spaces and proved the demiclosed principle for this class of mappings. A selfmap $T$ of a subset $E$ of a metric space is said to satisfy Condition $\left(B_{\gamma, \mu}\right)$ (or called Patir map) if there are some $\gamma \in[0,1]$ and $\mu \in[0$, $1 / 2]$ with $2 \mu \leq \gamma$ such that for all $a^{\prime}, a^{\prime \prime} \in E$,

$$
\begin{aligned}
\gamma \rho\left(a^{\prime}, T a^{\prime}\right) & \leq \rho\left(a^{\prime}, a^{\prime \prime}\right)+\mu \rho\left(a^{\prime \prime}, T a^{\prime \prime}\right) \Rightarrow \rho\left(T a^{\prime}, T a^{\prime \prime}\right) \\
& \leq(1-\gamma) \rho\left(a^{\prime}, a^{\prime \prime}\right)+\mu\left(\rho\left(a^{\prime}, T a^{\prime \prime}\right)+\rho\left(a^{\prime \prime}, T a^{\prime}\right)\right) .
\end{aligned}
$$


Iterative techniques for finding fixed points is very important and active research field of nonlinear analysis and has very fruitful applications in computers, applied economics, physics, and many applied sciences [15-26]. Since the Picard iteration $x_{m+1}=T x_{m}$ does not always converge to a fixed point of a given nonexpansive operator, we shall present here some other well known process originally due various reseachers, which are not only converges to fixed point of a given nonexpansive operator but also have better rate of convergence as compare Picard iteration. Let we assume $E$ be a nonempty as well as convex subset of a Banach space, $\alpha_{m}, \beta_{m} \in(0,1)$ and $T: E \rightarrow E$ be a given operator.

One of the earlier iteration process was defind by Mann [27] as follows:

$$
\left\{\begin{array}{l}
x_{1} \in E, \\
x_{m+1}=\left(1-\alpha_{m}\right) x_{m}+\alpha_{m} T x_{m}, m \geq 1,
\end{array}\right.
$$

The Ishikawa iteration process can be viewed as an extension of the Mann iteration, which was defined by Ishikawa in [28] as follows:

$$
\left\{\begin{array}{l}
x_{1} \in E \\
y_{m}=\left(1-\beta_{m}\right) x_{m}+\beta_{m} T x_{m} \\
x_{m+1}=\left(1-\alpha_{m}\right) x_{m}+\alpha_{m} T y_{m}, m \geq 1
\end{array}\right.
$$

Agarwal et al. [29] is the slightly modification of the Ishikawa iteration and was defined as follows:

$$
\left\{\begin{array}{l}
x_{1} \in E, \\
y_{m}=\left(1-\beta_{m}\right) x_{m}+\beta_{m} T x_{m}, \\
x_{m+1}=\left(1-\alpha_{m}\right) T x_{m}+\alpha_{m} T y_{m}, m \geq 1,
\end{array}\right.
$$

By [29], we know that Agarwal iterative process is much better than the earlier defined process, namely, Picard, Mann and Ishikawa iterative processes.

In the year 2016, Thakur et al. [30] suggested the below iterative process:

$$
\left\{\begin{array}{l}
x_{1} \in E \\
z_{m}=\left(1-\beta_{m}\right) x_{m}+\beta_{m} T x_{m}, \\
y_{m}=T\left(\left(1-\alpha_{m}\right) x_{m}+\alpha_{m} z_{m}\right), \\
x_{m+1}=T y_{m}, m \geq 1,
\end{array}\right.
$$

Thakur et al. [30] proved that the sequence $\left\{x_{m}\right\}$ defined by the iterative process (7) converges (under some approperiate situations) to a fixed point of a given Suzuki map. Moreover, they constructed a new example of Suzuki mappings $T$ and proved that the iterative process ( 7 ) converges faster to a fixed point $T$ as compared the earlier iterative processes due to Picard, Mann [27], Ishikawa [28], S [29], Noor [31] and Abbas [15].
Motivated by above, recently in 2020, Abedeljawad et al. [32] introduced a new iterative process, which they call it JA iteration process, as follows:

$$
\left\{\begin{array}{l}
x_{1} \in E \\
z_{m}=\left(1-\beta_{m}\right) x_{m}+\beta_{m} T x_{m}, \\
y_{m}=T z_{m}, \\
x_{m+1}=T\left(\left(1-\alpha_{m}\right) T x_{m}+\alpha_{m} T y_{m}\right), m \geq 1,
\end{array}\right.
$$

Abdeljawad et al. [32] establish that the sequence $\left\{x_{m}\right\}$ defined by the iterative process (8) converges (under some approperiate situations) to a fixed point of a given Patir map in Banach spaces. Moreover, they constructed a new example of Patir maps $T$ and proved that the iterative process (8) converges faster to a fixed point $T$ as compared the leadings iterative processes due to Agarwal [29] and Thakur et al. [30]. In this paper, we improve and extend their results to the nonlinear setting of Hadamard spaces.

\section{Preliminaries}

Throughout the sequel, we will write $\mathbb{P}$ and $\mathbb{R}$ for natural and real numbers sets, respectively. Assume that $(M, \rho)$ is a metric space. We can define a geodesic from $a^{\prime}$ to $a^{\prime \prime}$ as $g$ : [0, $c] \rightarrow \mathbb{R}$ which gives $g 0=a^{\prime}, g c=a^{\prime \prime}$ and $\rho\left(g l, g l^{\prime}\right)=\left|l-l^{\prime}\right|$ for every $l, l^{\prime} \in[0, m]$. In particular, the map $g$ is an isometry and $\rho\left(a^{\prime}, a^{\prime \prime}\right)=m$. The image of $g$ is known as a geodesic (or called metric segment) joining the element $a^{\prime}$ and $a^{\prime \prime}$. If any two elements of $M$ are connected by a geodesic then the metric space $(M, \rho)$ is called a geodesic space. If one have only one geodesic joining $a^{\prime}$ and $a^{\prime \prime}$ for each $a^{\prime}, a^{\prime \prime} \in M$ then it is called uniquely geodesic, which we often represent by $\left[a^{\prime}, a\right.$ ''] called the segment joining $a^{\prime}$ to $a^{\prime \prime}$.

Definition 1. Assume that $a, b_{1}, b_{2} \in M$ and $b_{0}$ is the midpoint of the segment $\left[b_{1}, b_{2}\right]$ such that,

$$
\rho\left(a, b_{0}\right)^{2} \leq \frac{1}{2} \rho\left(a, b_{1}\right)^{2}+\frac{1}{2} \rho\left(a, b_{2}\right)^{2}-\frac{1}{4} \rho\left(b_{1}, b_{2}\right)^{2} .
$$

Then (9) is known as the $C N$ inequality of Burhat and Tits [33].

A uniquely geodesic metric space $M$ is called $C A T(0)$ space if and only if $M$ is endowed with the $C N$ inequality (cf. [34]). A CAT(0) space is called Hadamard space if it is complete. For the detail study and results in Hadamard spaces, one can search $[34,35]$.

Definition 2. Take a bounded sequence $\left\{x_{m}\right\}$ in a Hadamard space $M$. Suppose $E$ is closed and convex in $M$. Fix $q \in M$, then we state the following.

$$
r\left(q,\left\{x_{m}\right\}\right)=\lim \sup _{m \rightarrow \infty} \rho\left(x_{m}, q\right)
$$

is known as the asymptotic radius of $\left\{x_{m}\right\}$ at $q$. 
The asymptotic radius of the sequence $\left\{x_{m}\right\}$ wrt $E$ is given by

$$
r\left(E,\left\{x_{m}\right\}\right)=\inf \left\{r\left(q,\left\{x_{m}\right\}\right): q \in E\right\} .
$$

Moreover, the set

$$
A\left(E,\left\{x_{m}\right\}\right)=\left\{q \in E: r\left(q,\left\{x_{m}\right\}\right)=r\left(E,\left\{x_{m}\right\}\right)\right\},
$$

is known as the asymptotic center of the sequence $\left\{x_{m}\right\}$ wrt to $E$.

Remark 3 . The cardinality of the set $A\left(E,\left\{x_{m}\right\}\right)$ in any Hadamard space is always equal to one, (see e.g., [36] and others).

The ([37], Proposition 2.1) tells us that in the setting of Hadamard spaces, for every bounded sequence, namely, \{ $\left.x_{m}\right\} \subseteq E$, the set $A\left(E,\left\{x_{m}\right\}\right)$ is essentially the subset of $E$ provided that $E$ is convex and bounded. It is well-known that \{ $\left.x_{m}\right\}$ has a subsequence which $\Delta$-converges to some point provided that the sequence is bounded.

Definition 4 (see [38]). A sequence $\left\{x_{m}\right\}$ in a given Hadamard space is said to be $\Delta$-convergent to $q \in M$ if and only if $q$ is the unique asymptotic center of the $\left\{u_{m}\right\}$. Where \{ $\left.u_{m}\right\}$ is any subsequence of the sequence $\left\{x_{m}\right\}$. We denote by $\Delta-\lim _{m} x_{m}=q$ and call the point $q$ the $\Delta-\lim$ of $\left\{x_{m}\right\}$.

Notice that a bounded sequence $\left\{x_{m}\right\}$ in a Hadamard space is known as regular if and only if for every subsequence, namely, $\left\{u_{m}\right\}$ of $\left\{x_{m}\right\}$ one has $r\left(\left\{x_{m}\right\}\right)=r\left\{u_{m}\right\}$. It is wellknown that, in the setting of Hadamard spaces each regular sequence $\Delta$-converges, and consequently each bounded sequence has a $\Delta$-convergent subsequence.

Definition 5 (see [37]). Let $T$ be a selfmap on a subset $E$ of a given Hadamard space and $f$ be a selfmap of $[0, \infty)$. We say that $T$ has condition $I$ if the following holds:

(i) $f(g)=0$ if and only if $g=0$

(ii) $f(g)>0$ for every $g>0$

(iii) $\rho\left(a^{\prime}, T a^{\prime}\right) \geq f\left(\rho\left(a^{\prime}, F_{T}\right)\right)$.

We now present some propositions and lemmas, which characterize the condition $(B \gamma, \mu)$.

Proposition 6 (see [14]). Suppose $E$ is a nonempty subset of a given Hadamard space M. If $T: E \rightarrow E$ has condition $(B \gamma, \mu)$. Then for every fixed point $w$ of $T$, one has

$$
\rho\left(w, T a^{\prime}\right) \leq \rho\left(w, a^{\prime}\right)
$$

for each $a^{\prime} \in E$.

Lemma 7 (see [14]). Suppose $E$ is nonempty closed convex subset of a given Hadamard space $M$. If $T: E \rightarrow E$ has $\left(B_{\gamma, \mu}\right)$ condition and the sequence $\left\{x_{m}\right\} \subseteq E$ satisfy $\lim _{m \rightarrow \infty} \rho\left(T x_{m}\right.$, $\left.x_{m}\right)=0$ and $\Delta-\lim _{m} x_{m}=w$, then $w=T w$.

Lemma 8 (see [39]). Let $E$ be a nonempty subset of a given Hadamard space $M$. If $T: E \rightarrow E$ has the $\left(B_{\gamma, \mu}\right)$ condition. Then the set $F_{T}$ always closed.

Lemma 9 (see [14], lemma 3.5). Suppose E be nonempty subset of a given Hadamard space $M$. If.

$T: E \rightarrow E$ has condition $\left(B_{\gamma, \mu}\right)$. Then for $a^{\prime}, a^{\prime \prime} \in E$ and $k \in[0,1]$, the following hold:

(i) $\rho\left(T a^{\prime}, T^{2} a^{\prime}\right) \leq \rho\left(a^{\prime}, T a^{\prime}\right)$,

(ii) either $\left(\left(h_{1}\right)\right.$ or $\left.\left(h_{2}\right)\right)$ satisfy:

$\left(h_{1}\right)(k / 2) \rho\left(a^{\prime}, T a^{\prime}\right) \leq \rho\left(a^{\prime}, a^{\prime \prime}\right)$

$\left(h_{2}\right)(k / 2) \rho\left(T a^{\prime}, T^{2} a^{\prime}\right) \leq \rho\left(T a^{\prime}, a^{\prime \prime}\right)$

(iii) $\rho\left(a^{\prime}, T a^{\prime \prime}\right) \leq(3-k+2 \mu) \rho\left(a^{\prime}, T a^{\prime}\right)+(1-k / 2) \rho\left(a^{\prime}\right.$ ,$\left.a^{\prime \prime}\right)+\mu\left(\rho\left(a^{\prime}, T a^{\prime \prime}\right)+\rho\left(a^{\prime \prime}, T a^{\prime}\right)+2 \rho\left(T a^{\prime}, T^{2} a^{\prime}\right)\right.$

Lemma 10 (see [40]). Let $M$ be a Hadamard space and $\left\{t_{m}\right\}$ be any real sequence such that $0<a \leq a_{m} \leq b<1$ for $m \geq 1$. Let $\left\{y_{m}\right\}$ and $\left\{z_{m}\right\}$ be any two sequences of $M$ such that $\lim \sup _{m \rightarrow \infty} \rho\left(y_{m}, x\right) \leq p, \lim \sup _{m \rightarrow \infty} \rho\left(z_{m}, x\right) \leq p \quad$ and $\lim _{m \rightarrow \infty} \rho\left(a_{m} y_{m} \oplus\left(1-a_{m}\right) z_{m}, x\right)=p$ hold for some $p \geq 0$. Then $\lim _{m \rightarrow \infty} \rho\left(y_{m}, z_{m}\right)=0$.

\section{Convergence Results for Mappings Satisfying $\left(B_{\gamma, \mu}\right)$ Condition}

This section establishes some important strong and $\Delta$-convergence results for operators endowed with the Condition $\left(B_{\gamma, \mu}\right)$.

Lemma 11. Let $E \neq \varnothing$ be a closed convex subset of a Hadamard space $M$ and $T: E \rightarrow E$ satisfies the $\left(B_{\gamma, \mu}\right)$ condition with $F_{T} \neq \varnothing$. If $\left\{x_{m}\right\}$ is a sequence generated by (8) (replacing + by $\oplus)$, then $\lim _{m \rightarrow \infty} \rho\left(x_{m}, w\right)$ exists for each $w \in F_{T}$.

Proof. Let $w \in F_{T}$. By Proposition 6, we have

$$
\begin{aligned}
\rho\left(z_{m}, w\right) & =\rho\left(\left(1-\beta_{m}\right) x_{m} \oplus \beta_{m} T x_{m}, w\right) \\
& \leq\left(1-\beta_{m}\right) \rho\left(x_{m}, w\right) \oplus \beta_{m} \rho\left(T x_{m}, w\right) \\
& \leq\left(1-\beta_{m}\right) \rho\left(x_{m}, w\right)+\beta_{m} \rho\left(x_{m}, w\right) \\
& \leq \rho\left(x_{m}, w\right),
\end{aligned}
$$

$$
\rho\left(y_{m}, w\right)=\rho\left(T z_{m}, w\right) \leq \rho\left(z_{m}, w\right) .
$$


They imply that

$$
\begin{aligned}
\rho\left(x_{m+1}, w\right) & =\rho\left(T\left(\left(1-\alpha_{m}\right) T x_{m} \oplus \alpha_{n} T y_{m}\right), w\right) \\
& \leq \rho\left(\left(1-\alpha_{m}\right) T x_{m} \oplus \alpha_{m} T y_{m}, w\right) \\
& \leq\left(1-\alpha_{m}\right) \rho\left(T x_{m}, w\right)+\alpha_{m} \rho\left(T y_{m}, w\right) \\
& \leq\left(\left(1-\alpha_{m}\right) \rho\left(x_{m}, w\right)+\alpha_{m} \rho\left(y_{m}, w\right)\right. \\
& \leq\left(\left(1-\alpha_{m}\right) \rho\left(x_{m}, w\right)+\alpha_{m} \rho\left(z_{m}, w\right)\right. \\
& \leq\left(\left(1-\alpha_{m}\right) \rho\left(x_{m}, w\right)+\alpha_{m} \rho\left(x_{m}, w\right)\right. \\
& =\rho\left(x_{m}, w\right) .
\end{aligned}
$$

Thus $\left\{\rho\left(x_{m}, w\right)\right\}$ is bounded below and nonincreasing and hence $\lim _{m \rightarrow \infty} \rho\left(x_{m}, w\right)$ exists for each $w \in F_{T}$.

Theorem 12. Suppose $E \neq \varnothing$ be a closed convex subset of a Hadamard space $M$. Assume that $T: E \rightarrow E$ satisfies the $\left(B_{\gamma, \mu}\right)$ condition. If $\left\{x_{m}\right\}$ is a sequence generated by (8) (replacing + by $\oplus$ ). Then, $F_{T} \neq \varnothing$ if and only if $\left\{x_{m}\right\}$ is bounded and $\lim _{m \rightarrow \infty} \rho\left(T x_{m}, x_{m}\right)=0$.

Proof. Suppose $F_{T} \neq \varnothing$ and $w \in F_{T}$. Then, by Lemma 11, $\lim _{m \rightarrow \infty} \rho\left(x_{m}, w\right)$ exists and $\left\{x_{m}\right\}$ is bounded. Put

$$
\lim _{m \rightarrow \infty} \rho\left(x_{m}, w\right)=p
$$

By the proof of Lemma 11 and (16), we have

$$
\limsup _{m \rightarrow \infty} \rho\left(z_{m}, w\right) \leq \limsup _{m \rightarrow \infty} \rho\left(x_{m}, w\right)=p .
$$

By proposition 6, we have

$$
\limsup _{m \rightarrow \infty} \rho\left(T x_{m}, w\right) \leq \limsup _{m \rightarrow \infty} \rho\left(x_{m}, w\right)=p .
$$

Also by the proof of Lemma 11, we have

$$
\rho\left(x_{m+1}, w\right) \leq\left(1-\alpha_{m}\right) \rho\left(x_{m}, w\right)+\alpha_{m} \rho\left(z_{m}, w\right)
$$

It follows that,

$$
\begin{aligned}
\rho\left(x_{m+1}, w\right)-\rho\left(x_{m}, w\right) \leq & \frac{\rho\left(x_{m+1}, w\right)-\rho\left(x_{m}, w\right)}{\alpha_{m}} \\
& \leq \rho\left(z_{m}, w\right)-\rho\left(x_{m}, w\right) .
\end{aligned}
$$

Therefore

$$
\begin{gathered}
p \leq \liminf _{m \rightarrow \infty} \rho\left(z_{m}, w\right) . \\
p=\lim _{m \rightarrow \infty} \rho\left(z_{m}, w\right)=\lim _{m \rightarrow \infty} \rho\left(\left(1-\beta_{m}\right) x_{m} \oplus \beta_{m} T x_{m}, w\right)
\end{gathered}
$$

Applying Lemma 10, we obtain

$$
\lim _{m \rightarrow \infty} \rho\left(T x_{m}, x_{m}\right)=0 .
$$

Conversely, let $w \in A\left(E,\left\{x_{m}\right\}\right)$. By Lemma 9(iii) for $\gamma=$ $k / 2, k \in(0,1)$, we have

$$
\begin{aligned}
\rho\left(x_{m}, T w\right) \leq & (3-k+2 \mu) \rho\left(x_{m}, T x_{m}\right)+\left(1-\frac{k}{2}\right) \rho\left(x_{m}, w\right) \\
& +\mu\left(\rho\left(x_{m}, T w\right)+\rho\left(w, T x_{m}\right)+2 \rho\left(T x_{m}, T^{2} x_{m}\right)\right)
\end{aligned}
$$

So by Proposition 6 and Lemma 9(i), we get

$$
\begin{aligned}
\rho\left(x_{m}, T w\right) \leq & (3-k+4 \mu) \rho\left(x_{m}, T x_{m}\right) \\
& +\left(1-\frac{k}{2}+\mu\right) \rho\left(x_{m}, w\right)+\mu \rho\left(x_{m}, T w\right)
\end{aligned}
$$

Then we have

$\rho\left(x_{m}, T w\right) \leq \frac{3-k+4 \mu}{1-\mu} \rho\left(x_{m}, T x_{m}\right)+\frac{1-(k / 2)+\mu}{1-\mu} \rho\left(x_{m}, w\right)$

This implies that

$$
\begin{aligned}
r\left(x_{m}, T w\right) & =\limsup _{m \rightarrow \infty} \rho\left(x_{m}, T w\right) \\
& \leq \frac{1-(k / 2)+\mu}{1-\mu} \limsup _{m \rightarrow \infty} \rho\left(x_{m}, w\right) \\
& \leq \limsup _{m \rightarrow \infty} \rho\left(x_{m}, w\right)=r\left(w, x_{m}\right)
\end{aligned}
$$

So $T w \in A\left(E,\left\{x_{m}\right\}\right)$. By the uniqueness of asymptotic centers, one can conclude that $T w=w$. This completes the proof.

The below stated and proved result establishes the $\Delta$ convergence for operators having condition $\left(B_{\gamma, \mu}\right)$ under $J A$ iterations in Hadamard spaces. This improves [[14], Theorem 4.3] in the sense of better rate of convergence.

Theorem 13. Suppose $E \neq \varnothing$ be a closed convex subset of $a$ Hadamard space $M$ and $T: E \rightarrow E$ be a mapping with $\left(B_{\gamma, \mu}\right)$ condition such that $F_{T} \neq \varnothing$. If $\left\{x_{m}\right\}$ is a sequence generated by (8) (replacing + by $\oplus$ ). Then $\left\{x_{m}\right\} \Delta$-converges to a fixed point of $T$.

Proof. By Theorem 12, the sequence $\left\{x_{m}\right\}$ is bounded. Hence one can take $A\left(\left\{x_{m}\right\}\right)=\{p\}$ for some $p \in M$. We are going to prove $A\left(\left\{x_{m_{k}}\right\}\right)=\{p\}$ for any subsequence $\left\{x_{m_{k}}\right\}$ of $\left\{x_{m}\right\}$. Suppose $\left\{x_{m_{k}}\right\}$ be a subsequence of $\left\{x_{m}\right\}$ such that $A\left(\left\{x_{m_{k}}\right\}\right)$ $=\{q\}$ Since $\left\{x_{m_{k}}\right\}$ is bounded, one can find a subsequence \{ $\left.x_{m_{j}}\right\}$ of $\left\{x_{m_{k}}\right\}$ such that $\left\{x_{m_{j}}\right\} \Delta$ converges to $w$ for some $w \in$ M. By Theorem 12, Lemma 7 one has $w \in F_{T}$ and hence $\lim _{m \rightarrow \infty} \sup \rho\left(x_{m}, w\right)$ exists. If $w \neq q$, then the singletoness of the cardinality of the asymptotic centers allows us the follwing 


$$
\begin{aligned}
\lim _{m \rightarrow \infty} \rho\left(x_{m}, w\right) & =\lim _{j \rightarrow \infty} \sup \rho\left(x_{m_{j}}, w\right)<\lim _{j \rightarrow \infty} \sup \rho\left(x_{m_{j}}, q\right) \\
& \left.\left.\leq \lim _{k \rightarrow \infty} \sup \rho\left(x_{m_{k}}, q\right)\right)<\lim _{k \rightarrow \infty} \sup \rho\left(x_{m_{k}}, w\right)\right) \\
& =\lim _{m \rightarrow \infty} \sup \rho\left(x_{m}, w\right)
\end{aligned}
$$

which is contradiction. Therefore, $q=w \in F_{T}$. Suppose that $p$ $\neq q$. Then

$$
\begin{aligned}
\lim _{m \rightarrow \infty} \rho\left(x_{m}, q\right) & =\lim _{k \rightarrow \infty} \sup \rho\left(x_{m_{k}}, q\right) \leq \lim _{k \rightarrow \infty} \sup \rho\left(x_{m_{k}}, p\right) \\
& \left.\left.\leq \lim _{m \rightarrow \infty} \sup \rho\left(x_{m}, p\right)\right)<\lim _{m \rightarrow \infty} \sup \rho\left(x_{m}, q\right)\right) \\
& =\lim _{m \rightarrow \infty} \sup \rho\left(x_{m}, q\right)
\end{aligned}
$$

$$
\left\{x_{m}\right\} \Delta \text {-converges to an element } p \in F_{T} \text {. }
$$

The follwing result establishes the strong-convergence for operators having condition $\left(B_{\gamma, \mu}\right)$ under $J A$ iterations in Hadamad spaces. We may notice that it is that analog of ([32], Theorem 20).

Theorem 14. Let $E \neq \varnothing$ be a closed convex subset of a Hadamard space $M$ and $T: E \rightarrow E$ be a map satisfying the $\left(B_{\gamma, \mu}\right)$. If $F_{T} \neq \varnothing$ and $\lim _{m \rightarrow \infty} \inf \rho\left(x_{m}, F_{T}\right)=0$, where $\left\{x_{m}\right\}$ be a sequence generated by $(8)$ (replacing + by $\oplus$ ). Then $\left\{x_{m}\right\}$ converges strongly to a fixed point of $T$.

Proof. By Lemma $11 \lim _{m \rightarrow \infty} \rho\left(x_{m}, w\right)$ exists for each $w \in F_{T}$. Thus $\lim _{m \rightarrow \infty} \rho\left(x_{m}, F_{T}\right)$ exists. Hence

$$
\lim _{m \rightarrow \infty} \rho\left(x_{m}, F_{T}\right)=0
$$

Hence one can find a subsequence $\left\{x_{m_{j}}\right\}$ of $\left\{x_{m}\right\}$ and \{ $\left.w_{j}\right\}$ in $F_{T}$ with $\rho\left(x_{m_{j}}, w_{j}\right) \leq 1 / 2^{j}, j \geq 1$. In the view of proof of Lemma 11, one can observe that

$$
\rho\left(x_{m+1}, w_{j}\right) \leq \rho\left(x_{m_{j}}, w_{j}\right) \leq \frac{1}{2^{j}} .
$$

Next it is our purpose to show that the sequence $\left\{w_{j}\right\}$ form a Cauchy sequence in $F_{T}$. For this, we consider the following

$$
\begin{aligned}
\rho\left(w_{j+1}, w_{j}\right) & \leq \rho\left(w_{j+1}, s_{m_{j+1}}\right)+\rho\left(s_{m_{j+1}}, w_{j}\right) \\
& \leq \frac{1}{2^{j+1}}+\frac{1}{2^{j}} \leq \frac{1}{2^{j-1}} \rightarrow 0, \text { as } j \rightarrow \infty .
\end{aligned}
$$

The above limit showes that the sequence $w_{j}$ is a Cauchy sequence in the set $F_{T}$. By Lemma 8 , the set $F_{T}$ is closed. Hence $w_{j} \rightarrow q$ for some $q \in F_{T}$. By Lemma 11, $\lim _{m \rightarrow \infty} \rho\left(x_{m}\right.$ $, q)$ exists. So the proof is finished.
TABLE 1: Strong convergence of leading iterative processes under $\alpha_{m}=(m+1) /(m+2)^{1 / 7}, \beta_{m}=1 /(2 m+3)^{1 / 2}$ and $x_{1}=2.4$.

\begin{tabular}{lccc}
\hline & JA (8) & Thakur (7) & Agarwal (6) \\
\hline$x_{1}$ & 2.4 & 2.4 & 2.4 \\
$x_{2}$ & 2.0416408217 & 2.0738540099 & 2.1477080198 \\
$x_{3}$ & 2.0043996405 & 2.0145672849 & 2.0582691397 \\
$x_{4}$ & 2.0004723516 & 2.0029812936 & 2.0238503460 \\
$x_{5}$ & 2.0000513645 & 2.0006250001 & 2.0100000027 \\
$x_{6}$ & 2.0000056425 & 2.0001333000 & 2.0042656021 \\
$x_{7}$ & 2.0000006250 & 2.0000288043 & 2.0018434768 \\
$x_{8}$ & 2.0000000697 & 2.0000062889 & 2.0008049909 \\
$x_{9}$ & 2.0000000078 & 2.0000013847 & 2.0003545001 \\
$x_{10}$ & 2.0000000008 & 2.0000003078 & 2.0001572218 \\
$x_{11}$ & 2 & 2.0000000685 & 2.0000701497 \\
$x_{12}$ & 2 & 2.0000000153 & 2.0000314632 \\
$x_{13}$ & 2 & 2.0000000034 & 2.0000141763 \\
$x_{14}$ & 2 & 2.0000000007 & 2.0000064132 \\
$x_{15}$ & 2 & 2.0000000001 & 2.0000029118 \\
$x_{16}$ & 2 & 2 & 2.0000013263 \\
\hline
\end{tabular}

Now we establish the final result in this section, which is related to the condition $I$. We may notice that it is the analog of ([32], Theorem 21).

Theorem 15. Suppose $E \neq \varnothing$ be a closed convex subset of $a$ Hadamard space $M$. Assume that $T: E \rightarrow E$ be a map having condition $\left(B_{\gamma, \mu}\right)$ with $F_{T} \neq \varnothing$. If $\left\{x_{m}\right\}$ is a sequence generated by $(8)$ (replacing + by $\oplus$ ). Then $\left\{x_{m}\right\}$ converges to an element of $F_{T}$ provided that $T$ has condition $(I)$.

Proof. It follows from Theorem 12 the $\lim _{m \rightarrow \infty} \inf \rho\left(x_{m}, T\right.$ $\left.x_{m}\right)=0$. By condition $(I), \lim _{m \rightarrow \infty}$ inf $\rho\left(x_{m}, F_{T}\right)=0$. The conclusions follows from the Theorem 14 .

\section{Numerical Interpretation}

In this section, we are interested in the rate of convergence. We first construct a new example of a mapping $T$ as follows, which is Patir mapping but not Suzuki.

Example 16. Define an operator $T:[1,4] \rightarrow[1,4]$ as follows

$$
T a^{\prime}=\left\{\begin{array}{l}
\frac{a^{\prime}+2}{2} \text { if } a^{\prime} \neq 4 \\
2 \text { if } a^{\prime}=4 .
\end{array}\right.
$$

To show that $T$ is not Suzuki mapping let $a^{\prime}=35 / 10$, $a^{\prime \prime}=4$. We see that, $1 / 2 \rho\left(a^{\prime}, T a^{\prime}\right)=3 / 8<1 / 2=\rho\left(a^{\prime}, a^{\prime \prime}\right)$ but $\rho\left(T a^{\prime}, T a^{\prime \prime}\right)=3 / 4>\rho\left(a^{\prime}, a^{\prime \prime}\right)$. Thus $T$ does not satisfy condition $(C)$. Choose $\gamma=1$ and $\mu=1 / 2$, we prove that $T$ has the $\left(B_{1,1 / 2}\right)$ condition. 


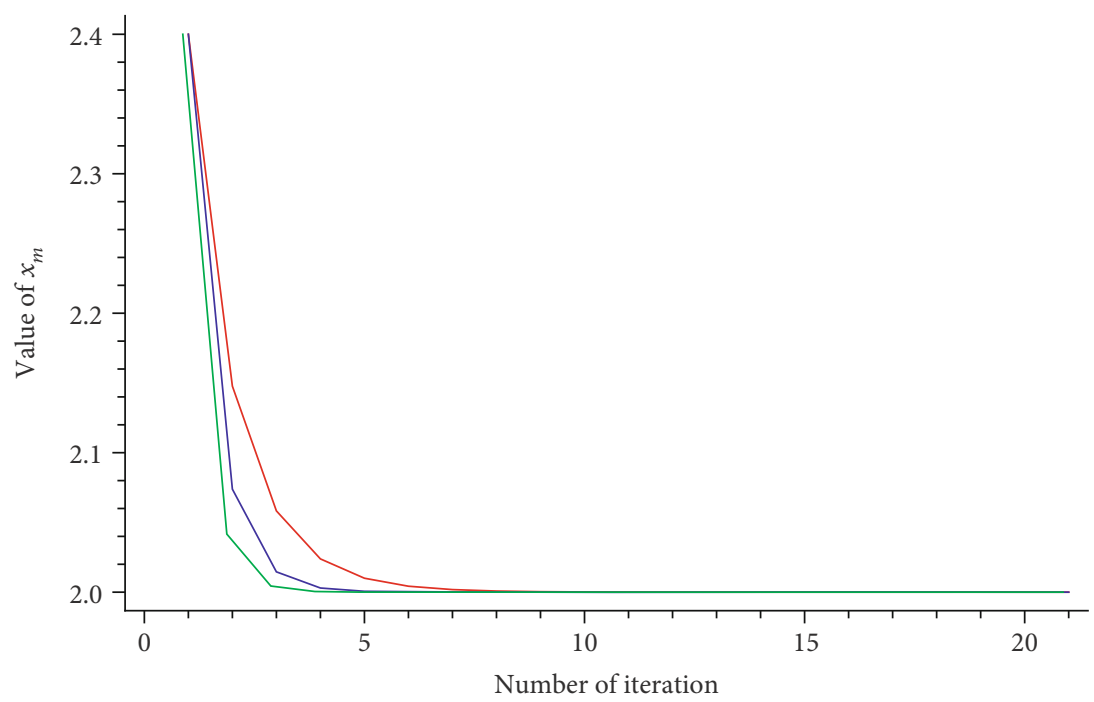

Figure 1: Convergence behaviors of JA (8), Thakur (7) and Agarwal (6) iterations for operator $T$ provided in the Example 16 under $x_{1}=2.4$.

$C_{1}:$ If we take $a^{\prime}, a^{\prime \prime} \in[1,4)$, then

$$
\begin{aligned}
(1-\gamma) & \rho\left(a^{\prime}, a^{\prime \prime}\right)+\mu\left(\rho\left(a^{\prime}, T a^{\prime \prime}\right)+\rho\left(a^{\prime \prime}, T a^{\prime}\right)\right) \\
= & \frac{1}{2}\left(\left|a^{\prime}-T a^{\prime \prime}\right|+\left|a^{\prime \prime}-T a^{\prime}\right|\right) \geq \frac{1}{2}\left|\frac{3 a^{\prime}}{2}-\frac{3 a^{\prime \prime}}{2}\right| \\
= & \frac{3}{4}\left|a^{\prime}-a^{\prime \prime}\right| \geq \frac{1}{2}\left|a^{\prime}-a^{\prime \prime}\right|=\rho\left(T a^{\prime}, T a^{\prime \prime}\right) .
\end{aligned}
$$

$C_{2}$ : If we take $a^{\prime} \in[1,4)$ and $a^{\prime \prime}=4$, then

$$
\begin{aligned}
(1-\gamma) & \rho\left(a^{\prime}, a^{\prime \prime}\right)+\mu\left(\rho\left(a^{\prime}, T b\right)+\rho\left(a^{\prime \prime}, T a^{\prime}\right)\right. \\
= & \frac{1}{2}\left(\left|a^{\prime}-T a^{\prime \prime}\right|+\left|a^{\prime \prime}-T a^{\prime}\right|\right) \\
= & \frac{1}{2}\left(\left|a^{\prime}-2\right|+\left|a^{\prime \prime}-\left(\frac{a^{\prime}+2}{2}\right)\right|\right) \\
= & \frac{1}{2}\left|a^{\prime}-2\right|+\frac{1}{2}\left|a^{\prime \prime}-\left(\frac{a^{\prime}+2}{2}\right)\right| \\
\geq & \frac{1}{2}\left|a^{\prime}-2\right|=\rho\left(T a^{\prime}, T a^{\prime \prime}\right) .
\end{aligned}
$$

$C_{3}$ : If we take $a^{\prime}=4=a^{\prime \prime}$, then we have

$$
\begin{aligned}
& (1-\gamma) \rho\left(a^{\prime}, a^{\prime \prime}\right)+\mu\left(\rho\left(a^{\prime}, T a^{\prime \prime}\right)+\rho\left(a^{\prime \prime}, T a^{\prime}\right)\right) \\
& \geq 0=\rho\left(T a^{\prime}, T a^{\prime \prime}\right) .
\end{aligned}
$$

Hence, $T$ satisfies the $\left(B_{1,1 / 2}\right)$ condition. The strong convergence of leading iterations can be seen in Table 1 and Figure 1 to the fixed point 2 of the mapping $T$. One can easly observe that JA iteration (8) converges faster than the leading Thakur (7) and leading Agarwal (6) iterative processes in Table 1 and Figure 1.

\section{Conclusions}

The study of fixed points in the framework of nonlinear domains gained much more attention of the mathematicians. Takahashi [41] was the first, who suggested the concept of convexity in metric spaces and proved some important fixed points results for nonexpansive operators in this setting. This convexity structure then initiated many other convexity structures in metric spaces. In this paper, we have established some strong and $\Delta$-convergence theorems for the class of Patir et al. [13] operators in nonlinear Hadamard spaces using new up-to-date iteration process which is faster than Picard, Mann, Ishikawa, S, Noor, Abbas and Thakur iterations. Moreover the class of Patir et al. [13] operators is more general than Suzuki operators and nonexpansive operators. Hence our results extend many known results of authors $[5-12,32]$ whose idea was limited to the setting of Suzuki operators.

\section{Data Availability}

No data were used to support this study.

\section{Conflicts of Interest}

We strongly declare that no one of us has conflicts of interest.

\section{Authors' Contributions}

K.U, J.A, A.A.K, M.d.I.S provided equal contributions to this article.

\section{Acknowledgments}

The authers are grateful to the Spanish Government for Grant RTI2018-094336-B-I00 (MCIU/AEI/FEDER, UE) and the Basque Government for Grant IT1207-19. 


\section{References}

[1] D. Gohde, “Zum Prinzip der Kontraktiven Abbildung," Mathematische Nachrichten, vol. 30, no. 3-4, pp. 251-258, 1965.

[2] W. A. Kirk, "Geodesic geometry and fixed point theory," in Seminar of mathematical analysis (Malaga/Seville, 2002/2003), vol. 64, pp. 195-225, Universidad de Sevilla, 2003.

[3] W. A. Kirk, "Geodesic geometry and fixed point theory II," Fixed Point Theory and Applications, 2004.

[4] T. Suzuki, "Fixed point theorems and convergence theorems for some generalized nonexpansive mappings," Journal of Mathematical Analysis and Applications, vol. 340, no. 2, pp. 1088-1095, 2008.

[5] S. Dhompongsa, W. Inthakon, and A. Kaewkhao, "Edelstein's method and fixed point theorems for some generalized nonexpansive mappings," Journal of Mathematical Analysis and Applications, vol. 350, no. 1, pp. 12-17, 2009.

[6] W. Phuengrattana, "Approximating fixed points of Suzukigeneralized nonexpansive mappings," Nonlinear Analysis: Hybrid Systems, vol. 5, no. 3, pp. 583-590, 2011.

[7] M. Basarir and A. Sahin, "On the strong and $\Delta$-convergence of $S$-iteration process for generalized nonexpansive mappings on CAT(0) spaces," Thai Journal of Mathematics, vol. 12, pp. 549-559, 2014.

[8] B. Nanjaras, B. Panyanak, and W. Phuengrattana, "Fixed point theorems and convergence theorems for Suzuki-generalized nonexpansive mappings in CAT(0) spaces," Nonlinear Analysis: Hybrid Systems, vol. 4, no. 1, pp. 25-31, 2010.

[9] K. Ullah and M. Arshad, "New iteration process and numerical reckoning fixed points in Banach spaces," University Politehnica of Bucharest Scientific Bulletin Series A, vol. 79, no. 4, pp. 113-122, 2017.

[10] I. Uddin and M. Imdad, "On certain convergence of S-iteration scheme in CAT(0) spaces," Kuwait Journal of Science, vol. 42, no. 2, pp. 93-106, 2015.

[11] I. Uddin and M. Imdad, "Convergence of SP-iteration for generalized nonexpansive mapping in Hadamard spaces," Hacettepe Journal of Mathematics and Statistics, vol. 47, no. 6, pp. 1595-1604, 2018.

[12] I. Uddin and M. Imdad, "Some convergence theorems for a hybrid pair of generalized nonexpansive mappings in CAT(0) spaces," Journal of Nonlinear and Convex Analysis, vol. 16, no. 3, pp. 447-457, 2015.

[13] B. Patir, N. Goswami, and V. N. Mishra, "Some results on fixed point theory for a class of generalized nonexpansive mappings," Fixed Point Theory and Applications, vol. 2018, no. 1, 2018.

[14] A. Varatechakongka and W. Phuengrattana, "A study on fixed point theorems for a class of generalized nonexpansive mappings in Hadamard spaces," Boletín de la Sociedad Matemática Mexicana, vol. 26, no. 2, pp. 687-699, 2020.

[15] M. Abbas and T. Nazir, "A new faster iteration process applied to constrained minimization and feasibility problems," Matematichki Vesnik, vol. 66, no. 2, pp. 223-234, 2014.

[16] Y. Yao, X. Qin, and J. C. Yao, "Projection methods for firmly type nonexpansive operators," Journal of Nonlinear and Convex Analysis, vol. 19, pp. 407-415, 2018.

[17] D. R. Sahu, F. Babu, and S. Sharma, "The S-iterative techniques on Hadamard manifolds and applications," Journal of Applied and Numerical Optimization, vol. 2, pp. 353-371, 2020.
[18] R. Fan, W. Wang, and C. Zhai, "Positive solutions for Hadamard fractional boundary value problems on an infinite interval," Journal of Nonlinear Functional Analysis, vol. 2020, no. 1, 2020.

[19] N. V. Hung, L. X. Dai, E. Kobis, and J. C. Yao, "The generic stability of solutions for vector quasi-equilibrium problems on Hadamard manifolds," Journal of Nonlinear and Variational Analysis, vol. 4, no. 3, pp. 427-438, 2020.

[20] P. Chuadchawna, A. Farajzadeh, and A. Kaewcharoen, "Convergence theorems of a modified iteration process for generalized nonexpansive mappings in hyperbolic spaces," Tohoku Mathematical Journal, vol. 72, no. 4, pp. 631647, 2020.

[21] P. Chuasuk, A. Farajzadeh, and A. Kaewcharoen, "An iterative algorithm for solving split feasibility problems and fixed point problems in p-uniformly convex and smooth Banach spaces," Journal of Computational Analysis and Applications, vol. 28, no. 1, pp. 49-66, 2020.

[22] A. Kaewcharoen, P. Chuadchawna, and A. Farajzadeh, "Fixed point approximations of generalized nonexpansive mappings via generalized M-iteration process in hyperbolic spaces," International Journal of Mathematics and Mathematical Sciences, vol. 2020, Article ID 6435043, 8 pages, 2020.

[23] P. Chuadchawna, A. Farajzadeh, and A. Kaewcharoen, "Convergence theorems for total asymptotically nonexpansive single-valued and quasi nonexpansive multivalued mappings in hyperbolic spaces," Journal of Applied Analysis, vol. 26, no. 1, pp. 117-130, 2020.

[24] T. Abdeljawad, K. Ullah, J. Ahmad, and N. Mlaiki, "Iterative approximations for a class of generalized nonexpansive operators in Banach spaces," Discrete Dynamics in Nature and Society, vol. 2020, Article ID 4627260, 6 pages, 2020.

[25] I. Uddin, J. Ali, and J. Nieto, "An iteration scheme for a family of multivalued mappings in CAT( 0 ) spaces with an application to image recovery," Revista de la Real Academia de Ciencias Exactas, Físicas y Naturales. Serie A. Matemáticas, vol. 112, no. 2, pp. 373-384, 2018.

[26] B. Panyanak, "The viscosity approximation method for multivalued G-nonexpansive mappings in Hadamard spaces endowed with graphs," Journal of Fixed Point Theory and Applications, vol. 22, no. 4, 2020.

[27] W. R. Mann, "Mean value methods in iteration," Proceedings of American Mathematical Society, vol. 4, no. 3, pp. 506-510, 1953.

[28] S. Ishikawa, "Fixed points by a new iteration method," Proceedings of American Mathematical Society, vol. 44, no. 1, pp. $147-150,1974$.

[29] R. P. Agarwal, D. O'Regan, and D. R. Sahu, "Iterative construction of fixed points of nearly asymptotically nonexpansive mappings," Journal of Nonlinear and Convex Analysis, vol. 8, no. 1, pp. 61-79, 2007.

[30] B. S. Thakur, D. Thakur, and M. Postolache, "A new iterative scheme for numerical reckoning fixed points of Suzuki's generalized nonexpansive mappings," Applied Mathematics and Computation, vol. 275, pp. 147-155, 2016.

[31] M. A. Noor, "New approximation schemes for general variational inequalities," Journal of Mathematical Analysis and Applications, vol. 251, no. 1, pp. 217-229, 2000.

[32] T. Abdeljawad, K. Ullah, and J. Ahmad, "Iterative algorithm for mappings satisfying $\left(\mathrm{B}_{\gamma, \mu}\right)$ condition," Journal of Function Spaces, vol. 2020, Article ID 3492549, 7 pages, 2020. 
[33] F. Bruhat and J. Tits, "Groupes Réductifs Sur Un Corps local," Publications Mathématiques de l'Institut des Hautes Études Scientifiques, vol. 41, no. 1, pp. 5-251, 1972.

[34] M. R. Bridson and A. Haefliger, Metric Spaces of Non-Positive Curvature, Vol. 319, Springer Science and Business Media, 2013.

[35] D. Burago, I. D. Burago, Y. Burago, S. A. Ivanov, and S. Ivanov, A Course in Metric Geometry, Vol. 33, American Mathematical Soc., 2001.

[36] S. Dhompongsa, W. A. Kirk, and B. Sims, "Fixed points of uniformly Lipschitzian mappings," Nonlinear Analysis: Theory, Methods \& Applications, vol. 65, no. 4, pp. 762-772, 2006.

[37] S. Dhompongsa, W. A. Kirk, and B. Panyanak, "Nonexpansive set-valued mappings in metric and Banach spaces," Journal of Nonlinear and Convex Analysis, vol. 8, no. 1, p. 35, 2007.

[38] S. Dhompongsa and B. Panyanak, "On $\triangle$-convergence theorems in CAT(0) spaces," Computers and Mathematics with Applications, vol. 56, no. 10, pp. 2572-2579, 2008.

[39] T. Abdeljawad, K. Ullah, and J. Ahmad, "On Picard-Krasnoselskii Hybrid Iteration Process in Banach Spaces," Journal of Mathematics, vol. 2020, Article ID 2150748, 5 pages, 2020.

[40] T. Laokul and B. Panyanak, "Approximating fixed points of nonexpansive mappings in CAT(0) spaces," International Journal of Mathematical Analysis, vol. 3, pp. 1305-1315, 2009.

[41] W. Takahashi, "A convexity in metric spaces and nonexpansive mappings," Kodai Mathematical Seminar Reports, vol. 2, pp. 142-149, 1970. 